Archives

13 | 1994

Enquêtes

\title{
La démarche comparative en histoire sociale
}

Sixième table-ronde franco-allemande d'histoire sociale (Merseburg, 5-7 mai 1994)

Hinnerk Bruhns

\section{(2) OpenEdition}

12 Journals

\section{Édition électronique}

URL : http://journals.openedition.org/ccrh/2714

DOI : $10.4000 /$ ccrh. 2714

ISSN : $1760-7906$

Éditeur

Centre de recherches historiques - EHESS

\section{Édition imprimée}

Date de publication : 4 octobre 1994

ISSN : 0990-9141

\section{Référence électronique}

Hinnerk Bruhns, "La démarche comparative en histoire sociale », Les Cahiers du Centre de Recherches Historiques [En ligne], 13| 1994, mis en ligne le 27 février 2009, consulté le 10 décembre 2020. URL: http://journals.openedition.org/ccrh/2714 ; DOI : https://doi.org/10.4000/ccrh.2714

Ce document a été généré automatiquement le 10 décembre 2020.

Article L.111-1 du Code de la propriété intellectuelle. 


\title{
La démarche comparative en histoire sociale
}

\author{
Sixième table-ronde franco-allemande d'histoire sociale (Merseburg, \\ 5-7 mai 1994)
}

Hinnerk Bruhns

1 Depuis 1987 les échanges intellectuels et la coopération scientifique entre spécialistes français et allemands de l'histoire sociale des $\mathrm{XIX}^{\mathrm{e}}$ et $\mathrm{XX}^{\mathrm{e}}$ siècles ont été rythmés par une série de rencontres bilatérales organisées sous l'égide de la Deutsche Forschungsgemeinschaft et du Centre National de la Recherche Scientifique : en 1987 à Lyon sur le thème "Itinéraires sociaux et professionnels dans l'entreprise: réalité et représentation ", en 1988 à Bad Homburg sur « La bourgeoisie en France et en Allemagne au XIX ${ }^{e}$ siècle ». La troisième table ronde a eu lieu à Arc et Senans en 1989 et était consacrée à «L'histoire sociale de la politique sociale de l'État en France et en Allemagne au XIX et au XXe siècles ». La quatrième table ronde portait sur le thème "Le monde des bureaux en France et en Allemagne aux XIX ${ }^{e}$ et Xx ${ }^{e}$ siècles " (Berlin 1991), et la cinquième a été organisée à Lyon en 1992 sur le thème « Stratégies patronales et marché du travail : entre mobilité et sédimentations ».

2 La sixième rencontre franco-allemande d'histoire sociale, qui a eu lieu à Merseburg en mai 1994, était consacrée à l'analyse de la démarche comparative dans des travaux en cours, comportant une comparaison entre la France et l'Allemagne, de la fin du XVIII ${ }^{e}$ au $\mathrm{xx}^{\mathrm{e}}$ siècle. Cette rencontre a été préparée comme les précédentes par Yves Lequin (Centre Pierre Léon, Lyon 11), Hartmut Kaelble (Université Humboldt de Berlin), Patrick Fridenson (EHESS), Heinz-Gerhardt Haupt (Université de Halle) et Hinnerk Bruhns (CNRS). L'organisation sur place à Merseburg a été assurée par Heinz-Gerhard Haupt, l'organisation générale par Hartmut Kaelble pour l'Allemagne et Hinnerk Bruhns pour la France. La rencontre a pu être menée à bien grâce au concours financier du CNRS, de la Deutsche Forschungsgemeinschaft, de la Mission Historique Française de Gottingen, ainsi que de l'Université Martin-Luther de Halle-Wittenberg.

3 Comme lors des rencontres antérieures, les textes, diffusés à l'avance, étaient en grande partie présentés par des historiens de la jeune génération et les commentaires 
introductifs aux débats, pour la plupart, par des historiens plus expérimentés. De jeunes politologues, sociologues et un linguiste ont également pris part à la table ronde. Pour la première fois aucune traduction n'était nécessaire, chacun s'exprimant dans la langue, le français ou l'allemand, qu'il maitrisait ou qu'il jugeait la plus appropriée à la situation. C'est un signe très encourageant qui montre que les efforts d'échanges et de formation de jeunes chercheurs à l'étranger entrepris notamment par la Mission Historique Française en Allemagne et le Programme Franco-Allemand du département Sciences de l'Homme et de la Société du CNRS portent leurs fruits.

4 La table ronde était organisée en quatre séances. Après une introduction générale sur l'usage de la comparaison en histoire (Hinnerk Bruhns), les communications de la première séance sur "Comparaisons nationales de politiques sociales et économiques " ont été surtout consacrées à l'action publique au niveau de l'État comme à celui des municipalités. Les sujets abordés avaient un point commun : la position des salariés dans la ville : comparaison des politiques sociales française et allemande de la deuxième moitié $\mathrm{du} \mathrm{XIX}^{\mathrm{e}}$ siècle (S. Kott, Poitiers) et de la Alterssicherung au début du $\mathrm{xx}^{\mathrm{e}}$ siècle (K. Schniedewind, Bremen), comparaison entre la Arbeitsvermittlung et le placement public avant 1914 (B. Zimmermann, Paris, et S. Rudischhauser, Freiburg), comparaison des politiques de transports urbains à Paris et Berlin entre 1890 et 1914 (E. Bendikat, Berlin). Le rapport introductif de Christoph Conrad (Berlin) insista sur la distinction entre deux niveaux de la comparaison. Le premier consiste en une comparaison "concrète", " réelle ", où les acteurs se trouvent confrontés eux-mêmes à la comparaison de deux ou plusieurs sociétés et où cette comparaison peut influer le cours des choses. Il s'agit là souvent plus d'une histoire des relations entre deux sociétés que d'une comparaison. Le deuxième niveau est celui de la comparaison comme procédé analytique et descriptif, et c'est celui sur lequel les recherches actuelles ont encore des progrès à faire.

La deuxième séance avait pour objet des « Comparaisons d'institutions, de pratiques et de mouvements sociaux ». R. Cayla (Lyon/Berlin) présenta un travail sur la formation professionnelle des ouvriers en Allemagne et en France entre 1918 et 1945, J. Vogel (Berlin) une comparaison des fêtes militaires et du culte de l'armée entre 1871 et 1914, et A. Wirsching (München) une comparaison des mouvements communistes à Berlin et à Paris entre 1919 et 1933/39. L'évolution actuelle des sociétés allemande et française fut abordée par C. de Galembert (Paris/Berlin) qui compara les attitudes de l'Église catholique en France et en Allemagne à l'égard des musulmans. Dans leurs rapports introductifs, Sylvie Schweitzer van de Casteele et Isabelle von Bultzingsloewen (Lyon) montrèrent que la comparaison, symétrique ou asymétrique, pouvait servir à tester des hypothèses comme celle d'un Sonderweg allemand, mais aussi que la comparaison de deux phénomènes historiques, dans deux cadres nationaux ou sociaux différents, ne peut constituer en elle-même une problématique scientifique suffisante et une légitimation de la comparaison dont la fonction heuristique doit donc être explicitée.

6 La troisième séance, "Comparaisons à base de prosopographies et études d'élites ", permit l'analyse de groupes sociaux très divers, allant de la chevalerie immédiate rhénane au XVII et XVIII ${ }^{e}$ siècles (C. Duhamelle, Gottingen), des négociants de Bordeaux, Hambourg et Libourne à l'époque napoléonienne ( $S$. Marzagalli, Mayence) et des richterliche Amtstrager à Amiens et à Kassel (C. Aumuller et S. Brakensiek, Bielefeld) aux professeurs d'université à Paris et Berlin (C. Charle, Paris) ou aux élites industrielles française et allemande après 1945 (H. Joly, Poitiers). Le rapport introductif de Hannes Siegrist (Berlin) mit en évidence la manière dont la comparaison de groupes sociaux et de 
biographies collectives aboutit à la constitution de types et de typologies et permet, sous l'angle d'une question spécifique, comme celle des conséquences sociales d'évènements politiques majeurs, d'établir des échelles pour la comparaison. Il fit part de son étonnement devant les réticences de certains historiens à se référer à des positions théoriques ou méthodologiques générales et à introduire un tertium comparationis. L'exposé de Christophe Duhamelle sur la chevalerie rhénane illustra l'utilité du recours à des typologies et à des comparaisons ciblées pour l'analyse d'un groupe social particulier et de ses comportements d'alliance. Le recours explicite à une comparaison (que Duhamelle appelle « une attitude comparatiste partielle ) évite ainsi « la juxtaposition de deux choix arbitraires et le simple inventaire des spécificités» et permet de «mieux comprendre selon quelles lignes de forces on peut essayer d'appréhender et de mettre en valeur la spécificité d'une élite régionale dans un Saint-Empire moderne dont la diversité et la complexité contraignent à une utilisation prudente des mises en parallèles ».

7 La dernière séance était consacrée à des "Études de l'espace public et analyses de discours ", avec les contributions de Martin Hummel (Marburg) sur le langage comme indicateur de processus sociaux, d'Axel Korner (Florence) sur la chanson ouvrière en France et en Allemagne au XIX ${ }^{e}$ siècle, de Jorg Requate (Berlin) sur la presse et l'évolution de la profession de journaliste en France et en Allemagne au XIX ${ }^{e}$ siècle, et d'Alexander Schmidt (Berlin) sur la perception de la bourgeoisie allemande dans une comparaison internationale. Dans son rapport introductif, Frédéric Barbier (EPHE) insista sur la difficulté, dans une démarche comparative axée sur les concepts et l'analyse de discours, à ajuster le choix de la perspective (Etats-nations/sociétés industrielles, etc.) et à saisir les rapports entre les changements de cadres géographiques ou géopolitiques et le changement de logiques chronologiques.

8 La présentation des rapports et des exposés a été organisée de façon à ce que les discussions portent plus sur la méthode, les problèmes et les expériences de la comparaison que sur les thèmes traités par les auteurs des communications. Ce principe ne fut, naturellement, pas toujours facile à mettre en pratique, tant il est vrai que l'intérêt de l'historien se porte naturellement en première ligne sur l'objet de sa recherche et non sur la méthode qu'il emploie. Il s'avéra que des comparaisons à base prosopographique ou des comparaisons d'institutions ne semblent, de prime abord, pas trop poser de problèmes méthodologiques. La comparaison de concepts, de discours, voire de "mentalités ", a été moins expérimentée dans la comparaison franco allemande mais semble prometteuse lorsqu'elle complète, comme dans le travail de Sabine Rudischhauser et Bénédicte Zimmermann sur Arbeitsvermittlung et placement public, des analyses d'institutions et de pratiques sociales. Plus complexes et moins prometteuses paraissent par contre des approches qui empruntent à certaines théories linguistiques ou à l'analyse de discours pour proposer un "tournant linguistique » à l'histoire sociale, comme le suggèrent certains auteurs américains.

9 La table ronde a confirmé qu'il est difficile d'aboutir, en histoire sociale comparée, à un consensus général sur l'utilisation de théories, notamment de théories empruntées à d'autres sciences sociales. Ce sujet a été très controversé lors de la table ronde. A ceux qui insistaient sur la nécessité de théories comme tertium comparationis s'opposaient ceux qui pensaient que ces théories sont trop liées à leur contexte d'origine et risquent de biaiser ou de rendre plus difficiles des comparaisons d'objets appartenant à d'autres contextes. Les historiens français présents à Merseburg se sont dans leur majorité montré moins enclins que leurs collègues allemands à recourir à des théories ou des modèles généraux 
et ont manifesté leur préférence pour la méthode inductive, progressant par généralisations successives. Mais plusieurs contributions mettent en évidence que de plus en plus d'historiens élaborent directement les cadres conceptuels et théoriques pour la comparaison. Les traditions différentes de l'histoire sociale dans les deux pays expliquent aisément que les références à des corpus comme, par exemple, celui de l'œuvre de Max Weber ou celui de Marc Bloch furent maniées de façon très différente par les participants français et allemands. L'idée qu'en histoire sociale comparée s'est développée, en interaction avec les sciences sociales, une tradition méthodologique et qu'il y existe un fonds de méthodes, d'instruments et d'expériences auquel l'historien peut légitimement recourir sans perdre son identité disciplinaire, cette idée n'est pas toujours facile à faire accepter.

Lors de cette table ronde, les objectifs même de la comparaison historique n'ont pu être discutés de façon systématique. Deux buts de la comparaison ont été cités souvent (il s'agit là d'aspects propres à la comparaison franco-allemande et qui ne peuvent être généralisés directement) l'un de ces buts de la comparaison est l'examen de représentations contemporaines d'un modèle allemand pour la France ou d'un modèle français pour l'Allemagne. Cet examen comparatif de modèles contemporains - qu'il s'agisse du modèle allemand de la formation universitaire, des taux de natalité élevés, de l'assurance sociale étatique, du développement de l'industrie lourde au XIX ${ }^{e}$ siècle, du modèle français de la révolution politique ou des prestations sociales communales aux $\mathrm{XVIII}^{\mathrm{e}}$ et $\mathrm{XIX}^{\mathrm{e}}$ siècles - ne vise pas à constater le retard ou l'avance d'un pays sur l'autre mais à mieux voir les spécificités et particularités d'un pays, comme dans le cas du système universitaire français analysé par Christophe Charle. En même temps l'historien est amené à s'interroger sur les relations entre les deux pays comparés. L'autre objectif de la comparaison, souvent cité dans les exposés et discussions, est de comprendre l'autre société à travers la comparaison de la langue contemporaine, des concepts, discours et mentalités. Par contre, l'analyse comparative en termes de différences de modernité ne semble plus à l'ordre du jour. L'idée d'un cheminement unilinéaire et ascendant des sociétés est abandonnée; ce qui se traduit d'ailleurs par le scepticisme envers des théories générales empruntées aux sciences sociales, évoqué plus haut. Les discussions ont néanmoins permis de réaffirmer l'utilité opérationnelle pour l'historien comparatiste de certaines théories, modélisations ou typologies développées dans les sciences sociales-et aussi en histoire!-et d'insister sur la nécessité d'un échange pluridisciplinaire plus approfondi sur la question de la comparaison.

11 La table ronde a donné l'impression qu'en histoire sociale européenne des $\mathrm{XIX}^{\mathrm{e}}$ et $\mathrm{xx}^{\mathrm{e}}$ siècles la comparaison franco-allemande est pratiquée de façon plus large et plus intensive que la comparaison de la France ou de l'Allemagne avec l'Italie, la GrandeBretagne ou d'autres pays. Parmi les raisons de cette priorité accordée à la comparaison franco-allemande il faut citer des raison historiques: les rivalités et conflits entre les deux pays avant 1945 et les relations étroites depuis les années 1950 et 1960 donnent de l'importance à la comparaison franco-allemande non seulement pour ces deux pays mais également pour l'Europe dans son ensemble. Une autre raison est apparemment l'attitude plus ouverte des jeunes historiens des deux pays qui, plus que ceux des générations précédentes, s'intéressent à l'histoire d'autres pays. Ainsi, il y a dix ou même cinq ans, il aurait été difficile d'organiser une table ronde réunissant autant de jeunes historiens français qu'allemands, parlant les deux langues et travaillant de façon comparative sur l'histoire sociale de leurs deux pays. 
12 A première vue il peut paraître paradoxal que la nation, comme unité de comparaison, ait été souvent au centre de cette table ronde franco-allemande; mais personne n'a considéré la nation comme une unité ou un cadre de comparaison naturel. La différenciation régionale des sociétés européennes ou à l'inverse le rôle particulier des capitales se traduit dans le fait qu'une grande partie des projets comparatifs francoallemands en cours sont des comparaisons au niveau régional ou local (sept projets parmi ceux présentés lors de la table ronde); cela vaut surtout pour les biographies collectives. D'autres projets montrèrent, au contraire, que des approches comparatives de problématiques historiques amènent souvent le chercheur à dépasser le cadre national qui paraît artificiel et a-historique. Parmi les exposés présentés, les analyses comparatives de discours butèrent le plus souvent aux frontières du national. Une autre question abordée à plusieurs reprises a été celle de savoir si, au moins au XIX ${ }^{e}$ siècle, l'État national, les institutions nationales et l'espace public national ne se trouvaient pas trop engagés dans un processus de formation pour pouvoir être pris sans grandes précautions comme un cadre pour des comparaisons internationales. D'autres arguments furent avancés pour la comparaison nationale : des modèles transnationaux ou des « retards » se situant pratiquement toujours à un niveau national, dans le cas allemand au moins au niveau germano-prussien.

13 Lors de la discussion finale, P. Fridenson insista sur le problème des échelles de comparaison (du local au transnational) et sur les effets d'interaction entre les phénomènes sociaux et leur traitement par l'historien. Parmi les questions et champs ouverts il releva la circulation des modèles (les transferts d'influences, etc.), le thème des cultures nationales et de l'effet sociétal, et, en définitive, l'interrogation sur l'influence de l'effondrement de la théorie des groupes sociaux. Cet effondrement va-t-il conduire les réflexions des chercheurs vers les milieux et les réseaux et les orienter vers des analyses comparatives sur le lien social? H. Kaelble de son côté releva l'augmentation considérable du nombre de travaux comparatifs francoallemands et la participation nombreuse de jeunes historiennes des deux pays. Quant aux motivations explicites des approches comparatives, il constata qu'elles sont plus souvent du côté de l'ouverture vers l'international par la comparaison et l'analyse de stéréotypes que du côté d'une approche anthropologique (dans le sens de la comparaison de comportements humains) ou de la nécessité de la construction d'une comparaison comme seule manière de comprendre un phénomène donné. $Y$. Lequin proposa de différencier entre trois démarches comparatives : 1) la comparaison terme à terme, appliquée à un sujet bien délimité ; 2) l'enquête comparative à grande échelle comme celle menée à Bielefeld sur les bourgeoisies européennes; 3) le comparatisme structurel qui utilise la comparaison à différents étapes de l'analyse historique (dont l'objet n'est pas forcément construit en terme de comparaison) comme un instrument heuristique.

14 La table ronde avait été organisée de manière à ce que tous les textes soient communiqués à l'avance aux participants et qu'ils soient présentés lors des séances par deux rapporteurs, un allemand et un français. Les discussions furent vives, voire passionnées. La relative unité spatiale et temporelle du champ exploré par les différentes comparaisons facilita certainement le dialogue (bilingue!) entre les participants. On aurait pu aussi espérer qu'elle permit d'obtenir plus facilement un consensus sur la pertinence (ou la non-pertinence) de telle ou telle approche comparative, plus facilement au moins que si on avait choisi des exemples très éloignés. Le rapporteur n'en est pas vraiment convaincu, car l'unité du champ entraîna souvent les discussions loin des 
préoccupations méthodologiques des organisateurs. Mais tous les participants acceptèrent de bonne grâce de se laisser ramener, si nécessaire, par les animateurs des séances sur le terrain aride de la réflexion méthodologique. 